Background Health systems in Africa which were mainly designed to manage acute communicable diseases based on a biomedical model now need to be able to handle the emerging non-communicable disease epidemic. Cardiovascular diseases such as heart failure are increasingly prevalent. Research from high income countries reveals that patients with heart failure have multidimensional needs especially a high symptom burden even when on drugs with a mortality benefit. We predict outcomes are likely to be even worse in African settings where access to these drugs is limited and poverty is rampant. Currently palliative care is mainly for HIV and cancer patients and has little impact on heart failure patients. In order to plan care based on needs it is necessary to elicit patients' perspectives of their needs and experiences of living with incurable illness.

Aim To understand the multidimensional experiences, needs, and use of services of patients living and dying of heart failure.

Methods In depth qualitative interviews are being conducted using the grounded theory approach with patients with heart failure recruited in Mulago hospital in Uganda. The interviews are supplemented by administering the African APCA POS questionnaire. Purposive sampling is being used for recruitment. Thematic saturation is expected to be achieved at a total of about 16 patients.

Results Preliminary analysis has identified the following themes: Lack of awareness of symptoms of heart failure which lead to late presentation, association of symptoms to witchcraft, significant distress from physical symptoms, recurrent admissions, increasing dependency associated with loss of dignity, loss of valued self, psychological distress, different theological ideas relating illness and the Divine and financial problems.

Conclusion Heart failure patients in Uganda have multidimensional needs some of which are similar to high income countries but others are unique to the African setting. Such patients could greatly benefit from a palliative approach.

\title{
Global Palliative Care
}

P 193 EXPERIENCE OF LIVING WITH ADVANCED HEART FAILURE IN UGANDA

Elizabeth Namukwaya, ${ }^{1,2}$ Scott Murray, ${ }^{1}$ Mhoira Leng, ${ }^{1,2,3}$ Julia Downing, ${ }^{2}$ Liz Grant'. 'University of Edinburgh, Edinburgh, United Kingdom; ${ }^{2}$ Makerere Palliative care unit, Department of Medicine Makerere University; ${ }^{3}$ Cairdeas International Palliative Care Trust 